\title{
European athletes dominate performances in Double Iron ultra-triathlons - A retrospective data analysis from 1985 to 2010
}

Rüst, Christoph Alexander ; Knechtle, Beat ; Knechtle, Patrizia ; Lepers, Romuald ; Rosemann, Thomas ; Onywera, Vincent

Abstract: We investigated the participation and performance trends of ultra-endurance triathletes from all nationalities competing in a Double Iron ultra-triathlon (7.6-km swim, 360-km cycle and 84.4-km run) from 1985 to 2010. A total of 1854 athletes participated in 92 Double Iron ultra-triathlons. The majority of the winners came from Europe with 72 victories, followed by North America with 17 victories. The race time for the European ultra-triathletes was 1340 ( $\mathrm{s}=95.3)$ min, decreasing highly significantly $(\mathrm{r} 2=0.28 ; \mathrm{P}<0.0001)$ across the years. North American ultra-triathletes finished the races within $1556(\mathrm{~s}=124.5) \mathrm{min}$; their race time showed no changes across the years (r $2=0.045 ; \mathrm{P}=0.07)$. The race time for the Europeans was highly significantly faster compared to the North Americans $(\mathrm{P}<0.0001)$. Future studies should investigate each country in Europe and North America in order to find the country with the largest participation of athletes and their best performance.

DOI: https://doi.org/10.1080/17461391.2011.641033

Posted at the Zurich Open Repository and Archive, University of Zurich ZORA URL: https://doi.org/10.5167/uzh-74027

Journal Article

Accepted Version

Originally published at:

Rüst, Christoph Alexander; Knechtle, Beat; Knechtle, Patrizia; Lepers, Romuald; Rosemann, Thomas; Onywera, Vincent (2014). European athletes dominate performances in Double Iron ultra-triathlons - A retrospective data analysis from 1985 to 2010. European Journal of Sport Science, 14(sup1):S39-S50.

DOI: https://doi.org/10.1080/17461391.2011.641033 


\section{European athletes dominate performances in Double Iron ultra-triathlons - a retrospective data analysis from 1985 to 2010}

Christoph Alexander Rüst ${ }^{1}$, Beat Knechtle ${ }^{1,2}$, Patrizia Knechtle ${ }^{2}$, Romuald Lepers ${ }^{3}$, Thomas Rosemann ${ }^{1}$,Vincent Onywera ${ }^{4,5}$

${ }^{1}$ Institute of General Practice and Health Services Research, University of Zurich, Zurich, Switzerland

${ }^{2}$ Gesundheitszentrum St. Gallen, St. Gallen, Switzerland

${ }^{3}$ INSERM U887, University of Burgundy, Faculty of Sport Sciences, Dijon, France

${ }^{4}$ Kenyatta University, Department of Recreation Management and Exercise Science, Kenya

${ }^{5}$ IAAF Athletics Academy at Kenyatta University, Kenya

\section{Corresponding author}

PD Dr. med. Beat Knechtle

Facharzt FMH für Allgemeinmedizin

Gesundheitszentrum St. Gallen

Vadianstrasse 26

9001 St. Gallen

Switzerland

Telefon

+41(0) 712268282

Telefax

+41(0) 712268272

e-mail:

beat.knechtle@hispeed.ch 


\section{Abstract}

We investigated the participation and performance trends of ultra-endurance triathletes from all nationalities competing in a Double Iron ultra-triathlon $(7.6 \mathrm{~km}$ swim, $360 \mathrm{~km}$ cycle, 84.4 $\mathrm{km}$ run) from 1985 to 2010 . A total of 1,854 athletes participated in 92 Double Iron ultratriathlons. The majority of the winners came from Europe with a total of 72 victories, followed by North America with 17 victories. The race time for the European ultra-triathletes was $1,340(s=95.3) \mathrm{min}$, decreasing highly significantly $\left(r^{2}=0.28 ; P<0.0001\right)$ across the years. North American ultra-triathletes finished the races within 1,556 $(s=124.5)$ min; their race time showed no changes across the years $\left(r^{2}=0.045 ; P=0.07\right)$. The race time for the Europeans was highly significantly faster compared with the North Americans $(P<0.0001)$. Future studies should investigate each country in Europe and North America in order to find the country with the largest participation of athletes and their best performance.

Key words: $\quad$ swimming - cycling - running - ultra-endurance 


\section{Introduction}

Triathlon is an endurance sport where the athletes have to compete in the three different endurance disciplines swimming, cycling and running. Competitions are held over different distances such as the Olympic distance over $1.5 \mathrm{~km}$ swim, $40 \mathrm{~km}$ cycle and $10 \mathrm{~km}$ run (Bernard, Sultana, Lepers, Hausswirth, \& Brisswalter, 2010; Sultana, Brisswalter, Lepers, Hausswirth, \& Bernard, 2008), the Ironman distance over $3.8 \mathrm{~km}$ swim, $180 \mathrm{~km}$ cycle, and 42.195 km run (Lepers, 2008; Lepers, \& Maffiuletti, 2011; Lepers, Sultana, Bernard, Hausswirth, \& Brisswalter, 2010), and then the ultra-distances, from the Double Iron ultratriathlon over $7.6 \mathrm{~km}$ swim, $360 \mathrm{~km}$ cycle, and $84.4 \mathrm{~km}$ run to the Deca Iron ultra-triathlon over $38 \mathrm{~km}$ swim, 1,800 km cycle, and $422 \mathrm{~km}$ run (Knechtle, Knechtle, \& Lepers, 2011a).

Regarding the performance in triathlons, the association of different characteristics in physiology (Laursen et al., 2005; O’Toole, 1989; O’Toole, Douglas, \& Hiller, 1989a; O’Toole, Douglas, \& Hiller, 1989b; Whyte et al., 2000), anthropometry (Knechtle, Knechtle, \& Rosemann, 2010a; Knechtle, Wirth, \& Rosemann, 2010b; Knechtle, Wirth, Baumann, Knechtle, \& Rosemann, 2010c), training (Gulbin, \& Gaffney, 1999; Knechtle, et al., 2010a; 2010b) and previous experience (Knechtle et al., 2010b; 2010c; Knechtle, Knechtle, Rosemann, \& Senn, 2011b; Rüst, Knechtle, Knechtle, Rosemann, \& Lepers, 2011) with race time have previously been investigated.

Apart from these aspects that might have a considerable influence on endurance performance, ethnicity and nationality may also have a substantial effect (Babel, Hertogh, \& Hue, 2005; Hamilton, 2000; Onywera, Scott, Boit, \& Pitsiladis, 2006; Onywera, 2009; Scott, et al., 2003; Scott, \& Pitsiladis, 2007). It is well known that Kenyan runners dominate distance running, 
such as in the marathon (Larsen 2003; Onywera, et al., 2006). Onywera et al. (2006) reported that Kenyan runners are from a distinctive environmental background in terms of geographical distribution and ethnicity, and that they also travelled further to school, mostly by running. These findings highlight the importance of environmental and social factors in the success of Kenyan runners. Apart from training (Larsen, 2003) and physiological factors (Larsen, 2003; Lucia, et al., 2006), environmental factors may also explain the dominance of Kenyan runners (Saltin, 1996; Onywera, et al., 2006; Onywera, 2009).

Considering the sports discipline triathlon, the 'Ironman Hawaii' was the first long-distance triathlon, held for the first time in 1978 (www.ironman.com/worldchampionship). Regarding the race results from 1978 to 2010, American triathletes dominated the 'Ironman Hawaii' in the beginning (Babbit, 2004), but now athletes from all over the world compete in this event. In 1985, in Huntsville (USA), US-American triathletes organised, for the first time, a longer triathlon consisting of twice the classic distance of the Ironman triathlon, named the Double Iron ultra-triathlon (Knechtle et al., 2011a). Over the 25-year period races of even longer distances, up to the Double Deca ultra-triathlon, have been organised around the world (www.iutasport.com).

To date, no study had investigated which countries ultra-triathlons were held in, and which nationalities had the most triathletes competing in, and winning, these races. Since the Double Iron ultra-triathlon was the first ultra-triathlon held over a longer distance than the 'Ironman Hawaii', we focused on races and athletes competing in Double Iron ultra-triathlons. Therefore, the aim of the present study was to investigate where Double Iron triathlons were held all over the world, and which nationalities compete in, and win, these races. Since these races were initiated and primarily held in the USA, we hypothesised that North-American triathletes would be among the best ultra-triathletes in Double Iron ultra-triathlons. 


\section{Methods}

The nationality, plus the swimming, cycling, running and total race times of all finishers in a Double Iron ultra-triathlon were analysed from 1985 to 2010 . The data set from this study was obtained from the race website of the International Ultra-Triathlon Association (www.iutasport.com) and from the Race Directors. This study was approved by the institutional review board of St. Gallen, Switzerland, with a waiver for the requirement of an informed consent, given that the study involved the analysis of publicly available data.

\section{Data analysis}

The performance of the athletes was expressed in minutes for the total race time and every split discipline. An analysis of the development of performance in total race times, as well as split times, was performed for the top three athletes for each year and each continent. Analyses could only be performed when at least three athletes from the regarded continent participated in a particular year. Therefore, it was not possible to analyse the development of performance for every year, every nation or every continent. Neither Africa nor Australia had three or more athletes participating in the same year and thus have been excluded from the analysis. For Asia, only in four years three or more athletes finished a Double Iron ultratriathlon successfully; from South America only in two years. Over the investigated 25 year period, a total of only 24 Asian athletes participated and 21 finished successfully. For South America, only 18 athletes started and 14 finished. Therefore, the analysis of the change in the yearly performance has been conducted only for North American and European triathletes and, subsequently, only these two continents were compared in the year-by-year analysis. For 24 out of the 25 years studied only European and North American triathletes provided sufficient available data for analysis. Due to the small amount of female participants (146 out 
of a total of 1,854 , equal to $~ 7.9 \%$ ), males and females were not analysed separately, but were pooled together in order to increase the sample size per continent.

\section{Statistical analysis}

Data were reported as means \pm standard deviation $(s)$ within the text. Linear regressions were used for estimating the changes of swimming, cycling, running and total times per year. Pearson's correlation coefficients were used to assess the association between variables. For comparisons between different groups, an unpaired $t$-test was used in case of two groups and one-way ANOVA with subsequent Tukey-Kramer post-hoc analysis in case of more than two groups. Statistical significance was accepted at $P<0.05$ (two-sided for t-tests). 


\section{Results}

\section{Participation}

In 1985, the first Double Iron ultra-triathlon was held in Huntsville, Alabama, USA (Table 1). This race was continued until 1996. After a break of one year, the Double Iron ultra-triathlon in the USA continued in Virginia. Regarding history, the first Double Iron ultra-triathlon in Europe was held in 1989 in Colmar, France. Over time, four races (Lévis in Canada, Monterrey in Mexico, Huntsville and Virginia in the USA) were held in North America. In South America, one race was held in Yahuarcocha, Ecuador. In Asia, one race was held in Ras-al-Khaimar in the United Arab Emirates. Most of the races were held, and are still held, in Europe. From 1985 to 2010, a total of 1,854 athletes participated in a Double Iron ultratriathlon, with 1,470 finishers and 384 non-finishers. On average, a total of $309(s=527)$ athletes from each continent (North America, South America, Europe, Africa, Asia and Australia) started, leading to a mean participation of $71(s=38)$ athletes per year. Table 1 shows the historical development of the 20 different competitions held over the Double Iron ultra-triathlon distance. Figure 1 shows the total number of participants per continent and year.

\section{Finishers and non-finishers}

Considering the performance of the athletes per continent, we found a mean of $245(s=427)$ finishers and $64(s=101)$ non-finishers, equal to $71(s=19) \%$ finishers. Regarding the whole time period, we detected $57(s=38)$ finishers per year and two non-finishers, corresponding to $80(s=7) \%$ finishers. Figure 2 shows the relationship of finishers and non-finishers for every year and all continents. Figure 3 presents the percentage of finishers for every continent and year. 


\section{Winners}

During the 25 investigated years, a total of 92 competitions were held, leading to 92 winners. No African, Australian or Asian ultra-triathlete recorded any victory. Most of the winners came from Europe, with a total of 72 victories, followed by North America and South America with 17 and 3 victories, respectively. Figure 4 shows the historical development of the total number of victories for North American, South American and European ultratriathletes over the 1985-2010 period. The first recorded win for a European ultra-triathlete was in 1988, but then the Europeans took over the leadership within two years and kept it until 2010.

\section{Performance in total race time}

For only two and four years, respectively, did at least three athletes participate from South America and Asia. Figure 5A shows the mean total race time in every year, as well as the linear regression, for the top three ultra-triathletes from Europe and North America. The mean total race time for the North American ultra-triathletes was 1,556 $(s=124.5)$ min, showing no changes over the studied period $\left(r^{2}=0.045 ; P=0.07\right)$. The mean total race time for the European ultra-triathletes was $1,340(s=95.3) \mathrm{min}$, but this time decreased significantly over the 25 -year period $\left(r^{2}=0.28 ; P<0.0001\right)$. The mean total race time of the North American and European ultra-triathletes was significantly different $(P<0.0001)$. In the year-by-year analysis, the European ultra-triathletes were significantly faster than the Americans in 1988, 1990-1992, 1998-2006 and 2009-2010 $(P<0.05)$. The American ultra-triathletes were never faster than the European ones. The mean total race time for the Asian ultra-triathletes was $1,774(s=141) \mathrm{min}$, this time was significantly slower than that for the North Americans or the Europeans $(P<0.05)$. The mean total race time for the South American ultra-triathletes was $1,578(s=87.2) \mathrm{min}$, this time was significantly slower than for the Europeans $(P<0.05)$ but not the North Americans. 


\section{Split time in swimming}

Figure 5B presents the mean swimming time, as well as the linear regression, for the top three athletes in every year for both the European and the North American ultra-triathletes. The swimming time for the North American ultra-triathletes was $121(s=21.9)$ min, showing no significant change over the studied period $\left(r^{2}=0.0025 ; P>0.05\right)$. The swimming time for European ultra-triathletes was $113.1(s=20.2) \mathrm{min}$, but this time decreased significantly over the 25 -year period $\left(r^{2}=0.19 ; P=0.0001\right)$. The swimming time of the North American ultratriathletes was no different from the swimming time of Europeans. In the year-by-year analysis, the American ultra-triathletes were significantly faster in 1991 and 1994, whereas the Europeans dominated in 1995, 1999, 2002-2005 and 2008-2009 $(P<0.05)$. The swimming time for the Asian ultra-triathletes was 174.9 (19.1) min, this time was significantly slower than for the North Americans or the Europeans $(P<0.05)$. The swimming time for the South American ultra-triathletes was $154.2(s=2.2)$ min, this time was significantly slower than for the Europeans $(P<0.05)$, but not the North Americans.

\section{Split time in cycling}

Figure 5C shows the mean cycling time and linear regression for the top three athletes in every year for both the Europeans and the North Americans. The cycling time for the European athletes was $676(s=34.3) \mathrm{min}$, but this time decreased significantly $\left(r^{2}=0.19 ; P<\right.$ 0.0001) over the years. In contrast, the cycling time for the North Americans remained unchanged at $737.1(s=43.6) \mathrm{min}$. The cycling times were significantly faster for the European ultra-triathletes than for the North Americans $(P<0.0001)$. In the year-by-year analysis, the European ultra-triathletes were significantly $(P<0.05)$ faster in 1990,1992 , 1998, 2001-2005 and 2009. The North American ultra-triathletes were never faster than the Europeans. The cycling time for the Asian and the South American ultra-triathletes was 877.3 
$(s=99.4)$ min and $830.6(s=14.8)$ min, respectively; these times were significantly slower than for the Europeans and the North Americans $(P<0.05)$.

\section{Split time in running}

The Figure 5D shows the mean running time and linear regression for the top three athletes in every year for both the Europeans and the North Americans. The mean running times for both the Europeans and the North Americans were equal at $496.5(s=71.2) \min$ and $615.7(s=$ 59.8) min, respectively. These times decreased significantly over the studied period $\left(r^{2}=\right.$ 0.26; $P<0.0001$ for the European athletes, and $r^{2}=0.12 ; P=0.002$ for the North American athletes, respectively). The running time was significantly $(P<0.0001)$ faster for the European ultra-triathletes compared with the North Americans. In the year-by-year analysis, the Europeans were significantly faster in 1988, 1990-1992, 1995-1996, 1998-2000, 2002 2006 and $2010(P<0.05)$. The North Americans were never faster than the Europeans. The running time for Asian ultra-triathletes was $704.9(s=84.1)$ min, this time was significantly slower than the European one $(P<0.05)$ but not the North American one. The running time for the South American ultra-triathletes was $591(s=112.2) \mathrm{min}$; this time was no different than that of the Europeans or the North Americans. 


\section{Discussion}

This is the first study to describe the historical development of the performance in ultraendurance triathletes competing in a Double Iron ultra-triathlon regarding their nationality. The North Americans were the first to organise a Double Iron ultra-triathlon in 1985, while the Europeans started in 1989 competing in these races. Since 1989, the number of races in Europe has greatly expanded, in contrast to the US-Americans who have continued to organise only one in their country. Also, in contrast to the North Americans, the Europeans have improved their performance over the studied period.

Regarding the history of triathlon, the first World Championship for the Olympic distance triathlon was held 1989 in Avignon (France), and the first World Championship for the longdistance triathlon was held in 1995 in Nice (France) (www.triathlon.org). For ultra-triathlons, most of the races over the Double Iron distance were held, and are still held, in Europe. However, regarding the continuity, a total of 14 different organisers offered races over this period in Europe. In North America, one Double Iron ultra-triathlon was almost continuously held in the USA. The Double Iron ultra-triathlon in Huntsville, Alabama, moved to Virginia, where, with a break of only one year, athletes have continued to be offered the possibility of competing in a Double Iron ultra-triathlon in the USA. Considering North America, only for a few years Double Iron ultra-triathlons were held in Canada and Mexico. For nine years, a Double Iron ultra-triathlon was held in Ecuador, South America. Regarding Europe, some Double Iron ultra-triathlons were held for only one year such as in France and Spain, while other races showed more continuity. The reasons for these findings might be that as only a small number of participants start in these races (Knechtle, et al., 2011a), presumably the expense of organising such a race was too high for organisers to continue offering them. This 
is in contrast to the USA where continuity at the race site in Huntsville, Alabama, and then Virginia was obvious, Double Iron ultra-triathlon races in Europe have moved from one site to another. Apart from the low number of participants and the costs of organising such a Double Iron ultra-triathlon, other reasons for this continuous relocating of the race sites must be considered. Following the IUTA (International Ultra-Triathlon Association) rules, the safety of the course is important (www.iutasport.com). Presumably, the European athletes and Race Directors were looking for safer race courses since these Double Iron ultra-triathlons last for more than 24 hours and the ultra-triathletes compete round the clock and sleep deprivation may increase the risk of accidents (Lucas, Anson, Palmer, Hellemans, \& Cotter, 2009). Another important finding was that no Double Iron ultra-triathlon was ever held in Australia, although, for the first time, Australia held a triathlon at the Sydney Olympics in 2000 (www.triathlon.org). Also, no Double Iron ultra-triathlon was ever held in Africa. We must assume that financial and social factors influence the organisation and participation in these ultra-endurance races.

Regarding the split performances, the North American triathletes were significantly faster in swimming in 1991 and 1994, whereas the European athletes dominated swimming in 1995, 1999, 2002-2005 and 2008-2009. It seems that the European triathletes improved their swimming performance, although the North American swimmers are among the best in the world (Pyne, Trewin, \& Hopkins, 2004; Trewin, Hopkins, \& Pyne, 2004). In cycling, the European ultra-triathletes improved their performance compared with the North Americans. Regarding running, both the North Americans and the Europeans improved their performance. The split discipline running seems to be the key discipline in an ultra-triathlon. It has been demonstrated in Triple Iron ultra-triathlons that a fast split time in running is more important than one in cycling (Knechtle, Duff, Amtmann, \& Kohler, 2007; Knechtle, \& Kohler, 2009). 
The improvement in the overall race time and split times in the European athletes, compared with the North Americans, is difficult to explain. A potential reason could be that winning an ultra-triathlon leads to more prestige in a European country than in North America. Another reason might be that there is a difference in the equipment and material available for triathletes in Europe and North America. Equipment such as wetsuits (Delextrat, Bernard, Hausswirth, Vercruyssen, \& Brisswalter, 2003) and triathlon specific race bikes (Garside \& Doran, 2000) have a considerable influence on race performance. Furthermore, training, race preparation and nutrition might also be different in these continents (Gulbin \& Gaffney, 1999; Knechtle et al., 2011a; O’Toole, 1989; Robins, 2007).

Apart from race equipment and nutrition, the fitness levels in a region or continent might also be important. Regarding the overall fitness levels between populations in the USA and Europe, a study in Poland showed a lower functional fitness in the Polish population than observed in the USA regarding elderly people from 65 to 94 years (Wiacek, \& Hagner, 2008). When the fitness levels of rural children in America and Russia were compared, Russian children showed a higher fitness level than their American counterparts (Hastie, Sinelnikov, \&Wadsworth, 2010). Olds, Tomkinson, Léger, and Cazorla (2006) investigated the worldwide variation in the performance of children and adolescents. They described a wide and significant global variability in their performance. The best performing children were from the Northern European countries such as Estonia, Iceland, Lithuania, and Finland. The worst performing children were from Singapore, Brazil, USA, Italy, Portugal, and Greece. There was evidence that performance was negatively related to being overweight, as well as to a country's average temperature. It was recently reported that body mass index in the general population was higher in the USA than in Europe (Finucane, et al., 2011). Kosti and Panagiotakos (2006) reported a dramatic increase in the prevalence of obesity in American children. 
A further explanation for both the higher participation and the faster race times of the Europeans might be the higher popularity of these races in Europe and among European athletes. Across the years, these events gained more popularity in Europe and in European athletes compared to the other continents. Also, we studied a very tiny segment of extremeendurance athletes - the top few who have the desire and ability to train for and complete these events. A recent study showed that 58 European athletes had already completed at least one Double Iron ultra-triathlon, one Triple Iron ultra-triathlon and one Deca Iron ultratriathlon, but only 11 North American ultra-triathletes managed to finish at least one race of each distance (Lepers, Knechtle, Knechtle, \& Rosemann, 2011). The present findings might simply reflect the differences in opportunity and interest in these races which is much greater in Europe than anywhere else in the world. The finding that the European athletes improved their ultra-triathlon performance should be examined and validated by analysing the historical development of performance in the first long-distance triathlon, the 'Ironman Hawaii'. In addition, the participation and performance in longer ultra-triathlon distances up to the Deca Iron ultra-triathlon should be investigated in detail. Also, a detailed analysis of both the participation and the performance of the European countries might give insights in which country the best European Double Iron ultra-triathletes live in and the possible reasons for their success in such events. Future studies investigating the motivation of ultra-endurance athletes to compete and finish these races might help to understand. It might also be of interest to investigate the trend of both North American and European athletes to travel to the other continent to compete in these races.

Furthermore, the motivation of these ultra-endurance athletes should be investigated as, presumably, different nationalities have different reasons. In Kenya, for example, Onywera et al. (2006) studied the demographic characteristics of elite Kenyan endurance runners. Kenyan runners were found to be from a distinctive environmental background in terms of 
geographical distribution, ethnicity and travelled further to and from school which was mainly by running. These findings underpin the importance of nurture, environmental and social factors in the success of Kenyan runners and are also indicative of the existence of some genetic advantage among Kenya which is yet to be unravelled. Regarding the motivation of Kenyan athletes to become a competitive athlete, both national (39\%) and international (34\%) athletes declared economic reasons. Typically, Kenyan athletes see running successes as means of making money to help their families, parents and siblings (Onywera, et al., 2006). The motivation of ultra-endurance athletes to train and compete in ultra-triathlons might be different and future studies investigating the motivation in these athletes might give more insights why they compete in these races.

\section{Conclusions}

To summarise, we found an increase in both the participation and the performance in European ultra-triathletes in Double Iron ultra-triathlons from 1985 to 2010. In contrast to the North American ultra-triathletes, the European athletes improved their overall performance as well as the performance in the three split-disciplines. Future studies should investigate how the European athletes participated and competed in the first long-distance triathlon, the 'Ironman Hawaii' or in other ultra-distance triathlons such as a Triple Iron ultra-triathlon or a Deca Iron ultra-triathlon. Future studies should also further investigate each country in Europe and North America in order to find the country with the largest participation of athletes and the best performance. It might be interesting to investigate the trend of both North American and European athletes to travel to the other continent to compete in these races. 


\section{References}

Babel, K., Hertogh, C., \& Hue, O. (2005). Influence of ethnic origin on predictive parameters of performance in sprint running in prepubertal boys. International Journal of Sports Medicine, 26, 798-802.

Babbitt, B. (2004). 25 years of the Ironman Triathlon World Championship. Oxford: Meyer \& Meyer; pp 190-196.

Bernard, T., Sultana, F., Lepers, R., Hausswirth, C., \& Brisswalter, J. (2010). Age-related decline in Olympic triathlon performance: effect of locomotion mode. Experimental Aging Research, 36, 64-78.

Delextrat, A., Bernard, T., Hausswirth, C., Vercruyssen, F., \& Brisswalter, J. (2003). Effects of swimming with a wet suit on energy expenditure during subsequent cycling. Canadian Journal of Applied Physiology, 28, 356-369.

Finucane, M. M., Stevens, A. G., Cowan, M. J., Danaei, G., Lin, J. K., Paciorek, C. J., Singh, G. M., Gutierrez, H. R., Lu, Y., Bahalim, A. N., Farzadfar, F., Riley, L. M., \& Ezzai, M. (2011). National, regional, and global trends in body-mass index since 1980: systematic analysis of health examination surveys and epidemiological studies with 960 country-years and 9.1 million participants. The Lancet, Early Online Publication, 4 February 2011, doi:10.1016/S0140-6736(10)62037-5

Garside, I., \& Doran, D. A. (2000). Effects of bicycle frame ergonomics on triathlon 10-km running performance. Journal of Sports Sciences, 18, 825-33.

Gulbin, J. P., \& Gaffney, P. T. (1999). Ultraendurance triathlon participation: typical race preparation of lower level triathletes. Journal of Sports Medicine and Physical Fitness, 39, $12-15$.

Hamilton, B. (2000). East African running dominance: what is behind it? British Journal of Sports Medicine, 34, 391-394.

Hastie, P., Sinelnikov, O., \& Wadsworth, D. (2010). Aerobic fitness status and out-of-school lifestyle of rural children in America and Russia. Journal of Physical Activity and Health, 7 , $150-155$

Knechtle, B., Duff, B., Amtmann, G., \& Kohler, G. (2007). Cycling and running performance, not anthropometric factors, are associated with race performance in a Triple Iron Triathlon. Research in Sports Medicine, 15, 257-269.

Knechtle, B., Knechtle, P., \& Lepers, R. (2011a). Participation and performance trends in ultra-triathlons from 1985 to 2009. Scandinavian Journal of Medicine and Science in Sports [Epub ahead of print]

Knechtle, B., Knechtle, P., Rosemann, T., \& Senn, O. (2011b). Personal best time, not anthropometry or training volume, is associated with total race time in a Triple Iron triathlon. The Journal of Strength and Conditioning Research [Epub ahead of print] 
Knechtle, B., Knechtle, P., \& Rosemann, T. (2010a). Similarity of anthropometric measures for male ultra-triathletes and ultra-runners. Perceptual and Motor Skills, 111, 805-818.

Knechtle, B., \& Kohler, G. (2009). Running performance, not anthropometric factors, is associated with race success in a Triple Iron Triathlon. British Journal of Sports Medicine, 43, 437-441.

Knechtle, B., Wirth, A., \& Rosemann, T. (2010b). Predictors of race time in male Ironman triathletes: physical characteristics, training or pre race experience? Perceptual and Motor Skills, 111, 437-446.

Knechtle, B., Wirth, A., Baumann, B., Knechtle, P., \& Rosemann, T. (2010c). Personal best time, percent body fat and training volume are differently associated with race time in male and female Ironman triathletes. Research Quarterly for Exercise and Sport, 81, 62-68.

Kosti, R. I., \& Panagiotakos, D. B. (2006). The epidemic of obesity in children and adolescents in the world. Central European Journal of Public Health, 14, 151-159.

Larsen, H. B. (2003). Kenyan dominance in distance running. Comparative Biochemistry and Physiology Part A: Molecular \& Integrative Physiology, 136, 161-170.

Laursen, P. B., Knez, W. L., Shing, C. M., Langill, R. H., Rhodes, E. C., \& Jenkins, D. G. (2005). Relationship between laboratory-measured variables and heart rate during an ultraendurance triathlon. Journal of Sports Sciences, 23, 1111-1120.

Lepers, R. (2008). Analysis of Hawaii ironman performances in elite triathletes from 1981 to 2007. Medicine and Science in Sports and Exercise, 40, 1828-1834.

Lepers, R., Knechtle, P., Knechtle, B., Rosemann, T. (2011). Analysis of ultra-triathlon performances. Open Access Journal of Sports Medicine, 2, 131-136.

Lepers, R., \& Maffiuletti, N. A. (2011). Age and gender interactions in ultraendurance performance: insight from the triathlon. Medicine and Science in Sports and Exercise, 43, 134-139.

Lepers, R., Sultana, F., Bernard, T., Hausswirth, C., \& Brisswalter, J. (2010). Age-related changes in triathlon performances. International Journal of Sports Medicine, 31, 251-256.

Lucas, S. J., Anson, J. G., Palmer, C. D., Hellemans, I. J., \& Cotter, J. D. (2009). The impact of 100 hours of exercise and sleep deprivation on cognitive function and physical capacities. Journal of Sports Sciences, 27, 719-728.

Lucia, A., Esteve-Lanao, J., Oliván, J., Gómez-Gallego, F., San Juan, A. F., Santiago, C., Pérez, M., Chamorro-Viña, C., \& Foster, C. (2006). Physiological characteristics of the best Eritrean runners-exceptional running economy. Applied Physiology, Nutrition, and Metabolism, 31, 530-540.

Olds, T., Tomkinson, G., Léger, L., \& Cazorla, G. (2006). Worldwide variation in the performance of children and adolescents: an analysis of 109 studies of the 20-m shuttle run test in 37 countries. Journal of Sports Sciences, 24, 1025-1038. 
Onywera, V. O., Scott, R. A., Boit, M. K., \& Pitsiladis, Y. P. (2006). Demographic characteristics of elite Kenyan endurance runners. Journal of Sports Sciences, 24, 415-422.

Onywera, V. O. (2009). East African runners: their genetics, lifestyle and athletic prowess. Medicine and Sport Science, 54, 102-109.

O'Toole, M. L. (1989). Training for ultraendurance triathlons. Medicine and Science in Sports and Exercise, 21, S209-S213.

O'Toole, M. L., Douglas, P. S., \& Hiller, W. D. (1989a). Applied physiology of a triathlon. Sports Medicine, 8, 201-225.

O'Toole, M. L., Douglas, P. S., \& Hiller, W. D. (1989b). Lactate, oxygen uptake, and cycling performance in triathletes. International Journal of Sports Medicine, 10, 413-438.

Pyne, D., Trewin, C., \& Hopkins, W. (2004). Progression and variability of competitive performance of Olympic swimmers. Journal of Sports Sciences, 22, 613-620.

Robins, A. (2007). Nutritional recommendations for competing in the Ironman triathlon. Current Sports Medicine Reports, 6, 241-248.

Rüst, C. A., Knechtle, B., Knechtle, P., Rosemann, T., \& Lepers, R. (2011). Personal best times in an Olympic distance triathlon and a marathon predict Ironman race time in recreational male triathletes. Open Access Journal of Sports Medicine, 2, 121-129.

Saltin, B. (1996). Exercise and the environment: Focus on altitude. Research Quarterly for Exercise and Sport, 67, S1-S10.

Scott, R. A., Georgiades, E., Wilson, R. H., Goodwin, W. H., Wolde, B., \& Pitsiladis, Y. P. (2003). Demographic characteristics of elite Ethiopian endurance runners. Medicine and Science in Sports and Exercise, 35, 1727-1732.

Scott, R.A., \& Pitsiladis, Y.P. (2007). Genotypes and distance running : clues from Africa. Sports Medicine, 37, 424-427.

Sultana, F., Brisswalter, J., Lepers, R., Hausswirth, C., \& Bernard, T. (2008). Effects of age and gender on Olympic triathlon performances. Science and Sports, 23, 130-135.

Trewin, C. B., Hopkins, W. G., \& Pyne, D. B. (2005). Relationship between world-ranking and Olympic performance of swimmers. Journal of Sports Sciences, 22, 339-345.

Whyte, G., Lumley, S., George, K., Gates, P., Sharma, S., Prasad, K., \& McKenna, W. J. (2000). Physiological profile and predictors of cycling performance in ultra-endurance triathletes. The Journal of Sports Medicine and Physical Fitness, 40, 103-109.

Wiacek, M., \& Hagner, W. (2008). The history and economic impact on the functional fitness of elderly in the South-Eastern region of Poland: a comparison with US citizens. Archives of Gerontology and Geriatrics, 46, 221-226. 


\section{Figure captions}

\section{Figure 1}

Total number of participants in a Double Iron ultra-triathlon, per continent and year, over the 1985-2010 period.

\section{Figure 2}

Panels A-F show the relationship of finishers and non-finishers in a Double Iron ultratriathlon, per continent and year, over the 1998-2010 period. Total bar lengths indicate the total number of participants per continent and year.

\section{Figure 3}

Panels A-F show the percentage of finishers in a Double Iron ultra-triathlon, per continent and year, over the 1985-2010 period.

\section{Figure 4}

Total number of wins in a Double Iron ultra-triathlon, per continent, over the 1985-210 period

\section{Figure 5}

Mean and linear regression of the total race time (Panel A) as well as the split times in swimming (Panel B), cycling (Panel C) and running (Panel D) of the top three North American and European athletes for every year over the 1985-2010 period. Data points represent mean values of the top three athletes per discipline or total race time. For North American athletes the split time in running changed significantly $\left(r^{2}=0.12 ; P=0.002\right)$, but neither the total race time, nor the split times in cycling or swimming. For European athletes the total race time $\left(r^{2}=0.28 ; P<0.0001\right)$ as well as the split time in swimming $\left(r^{2}=0.19 ; P=\right.$ $0.0001)$, running $\left(r^{2}=0.25 ; P<0.0001\right)$ and cycling $\left(r^{2}=0.19 ; P<0.0001\right)$ changed highly significantly over the 25 -year period. When the two continents were compared, European athletes dominated in all disciplines as well as in the total race time and, therefore, overwhelm the North American athletes. * indicate a significant difference in the respective year $(P<$ $0.05)$. 
Table 1: History of the races held in Double Iron ultra-triathlons

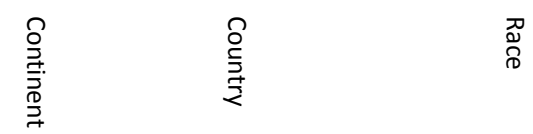

$\begin{array}{lll}\text { North America } & \text { Canada } & \text { Lévis } \\ \text { North America } & \text { Mexico } & \text { Monterrey } \\ \text { North America } & \text { USA } & \text { Huntsville } \\ \text { North America } & \text { USA } & \text { Virgina } \\ \text { South America } & \text { Ecuador } & \text { Yahuarcocha } \\ \text { Europe } & \text { Austria } & \text { Moosburg } \\ \text { Europe } & \text { Austria } & \text { Neulengbach } \\ \text { Europe } & \text { Belgium } & \text { Leuven } \\ \text { Europe } & \text { Belgium } & \text { Middelkerken } \\ \text { Europe } & \text { France } & \text { Colmar } \\ \text { Europe } & \text { France } & \text { Fontanil } \\ \text { Europe } & \text { Germany } & \text { Ottobrunn } \\ \text { Europe } & \text { Great Britain } & \text { Lichfield } \\ \text { Europe } & \text { Hungary } & \text { Bonyhad } \\ \text { Europe } & \text { Lithuania } & \text { Panevezys } \\ \text { Europe } & \text { Netherlands } & \text { Den Haag } \\ \text { Europe } & \text { Netherlands } & \text { Lelystad } \\ \text { Europe } & \text { Slovenia } & \text { Bakovic } \\ \text { Europe } & \text { Spain } & \text { Terrassa } \\ \text { Asia } & \text { United Arab Emirates } & \text { Ras-al-Khaimar }\end{array}$

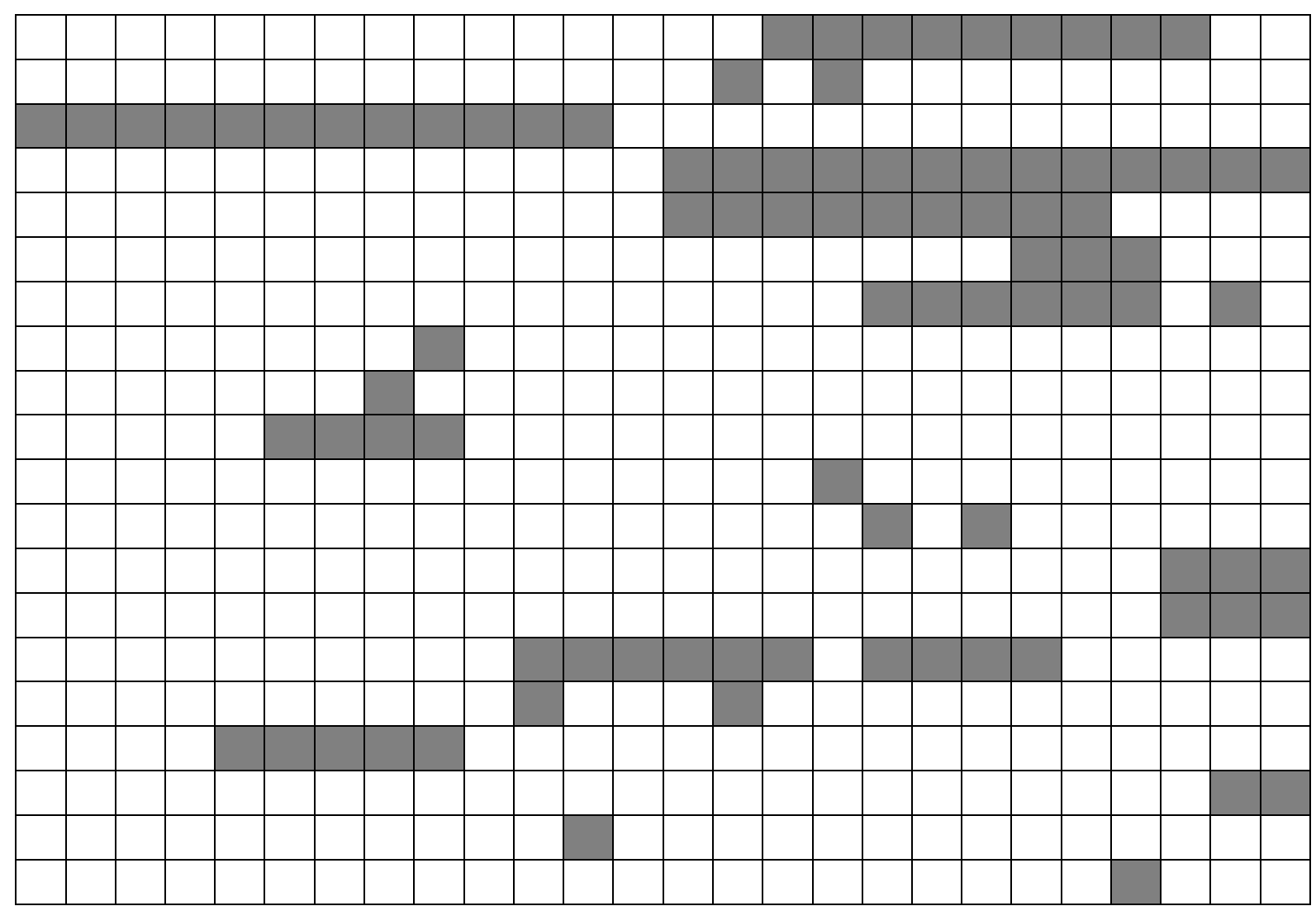




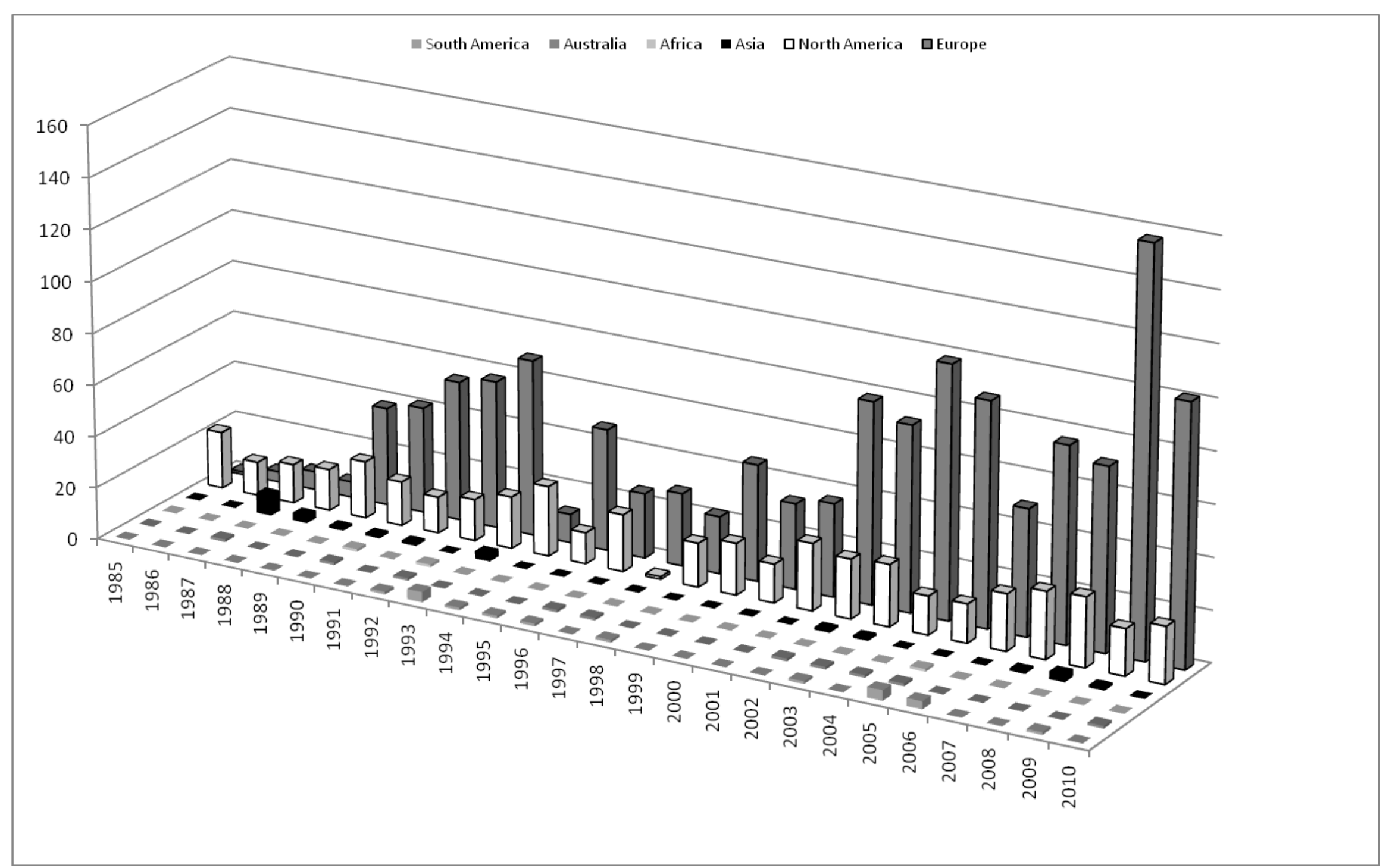

Figure 1 

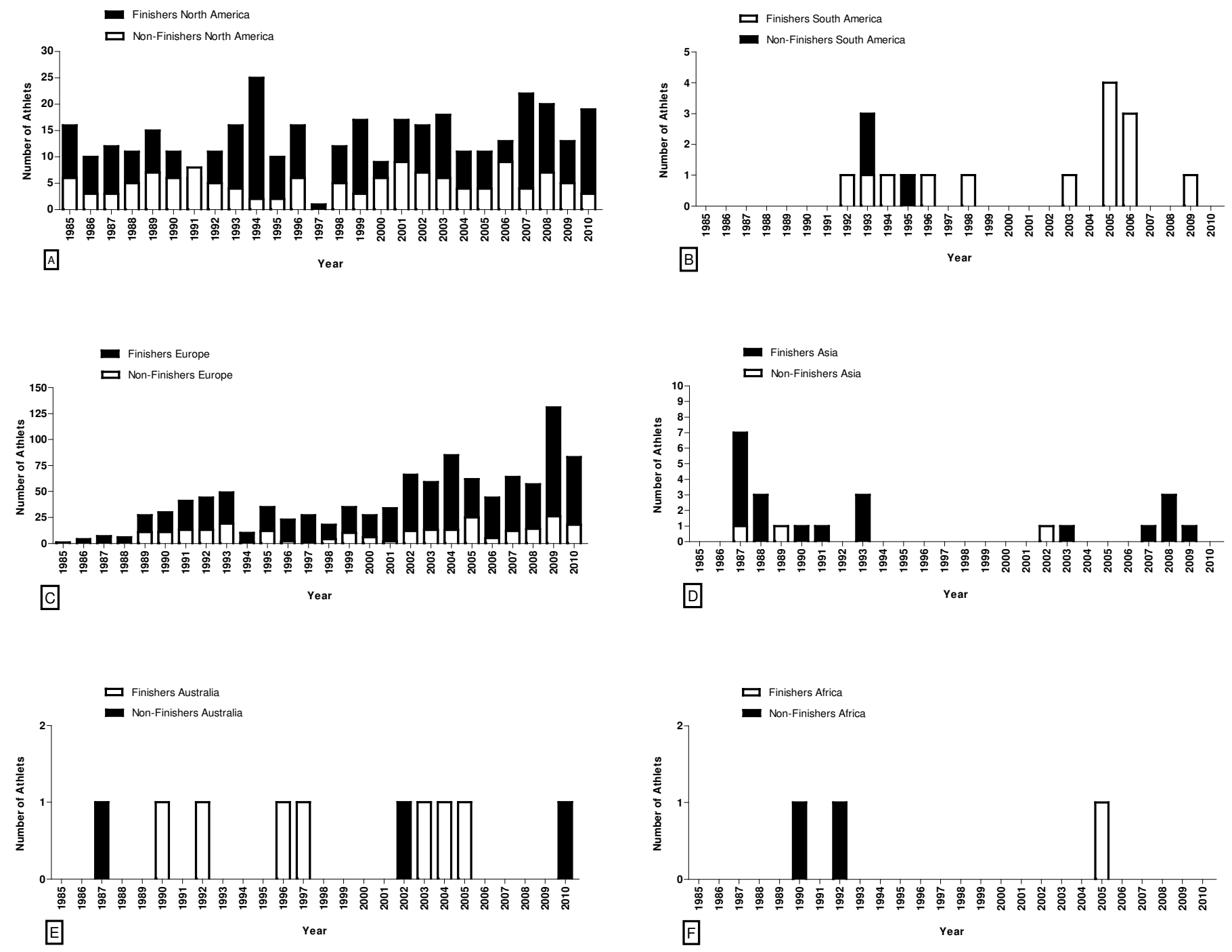

Figure 2 

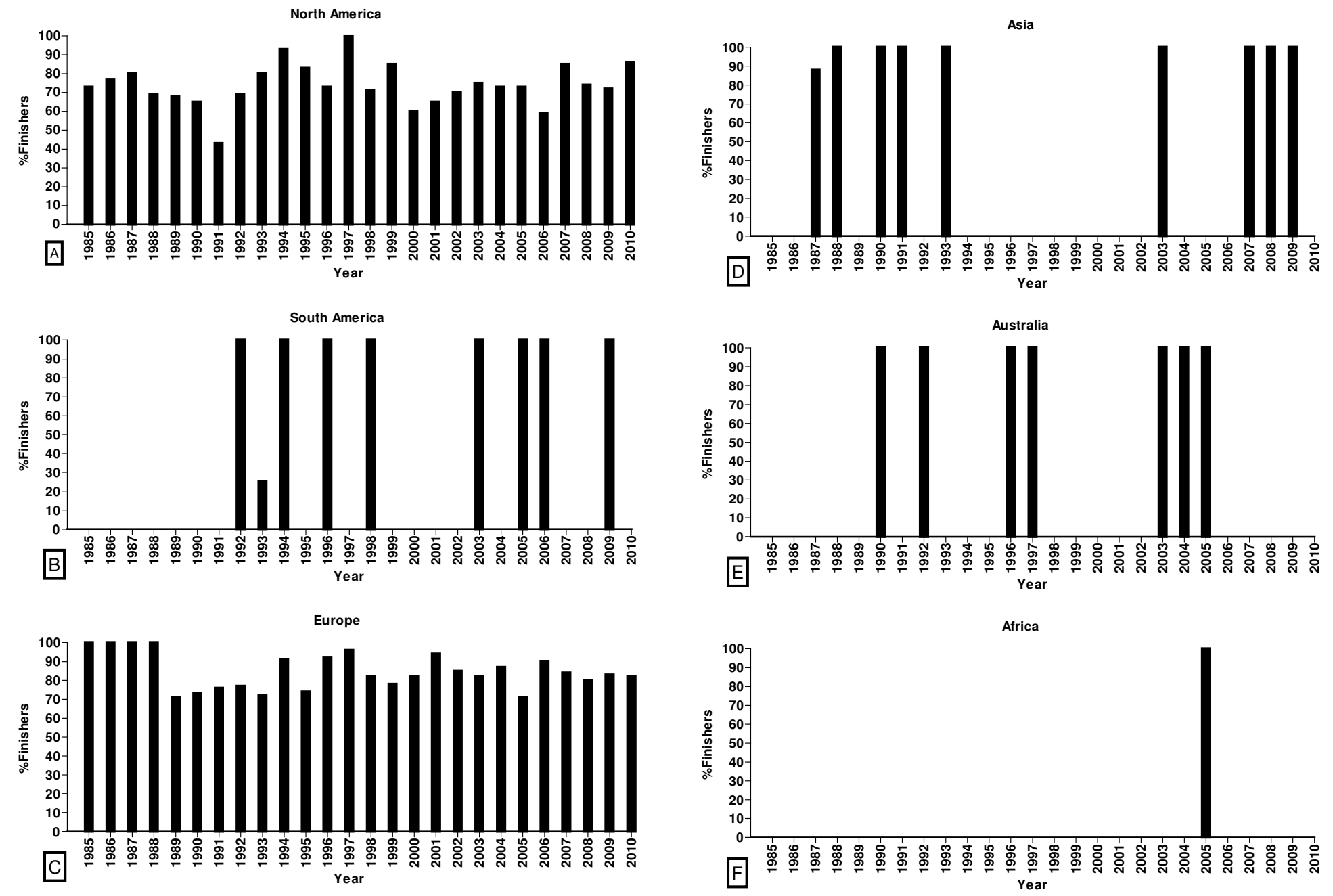

Figure 3 


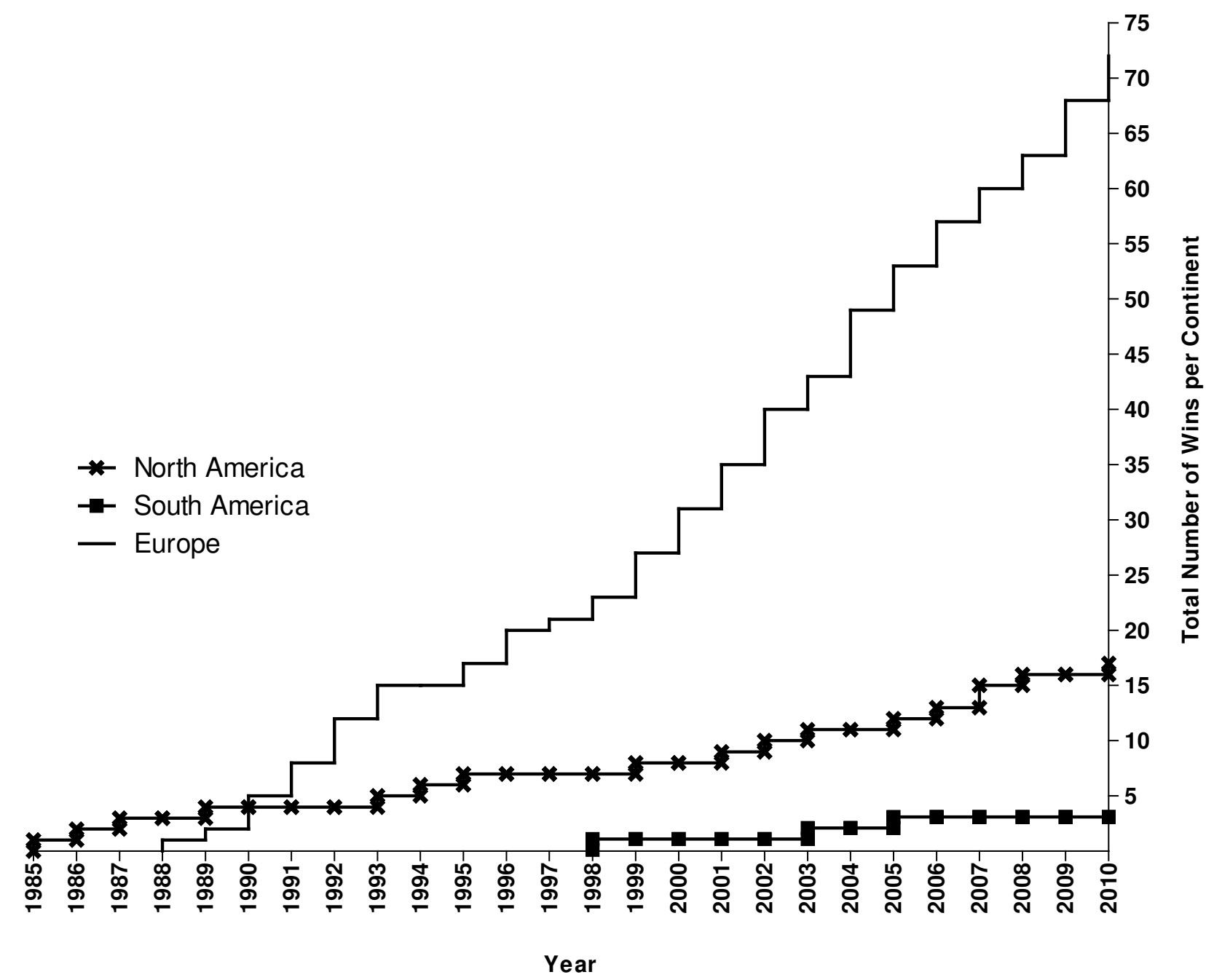

Figure 4 

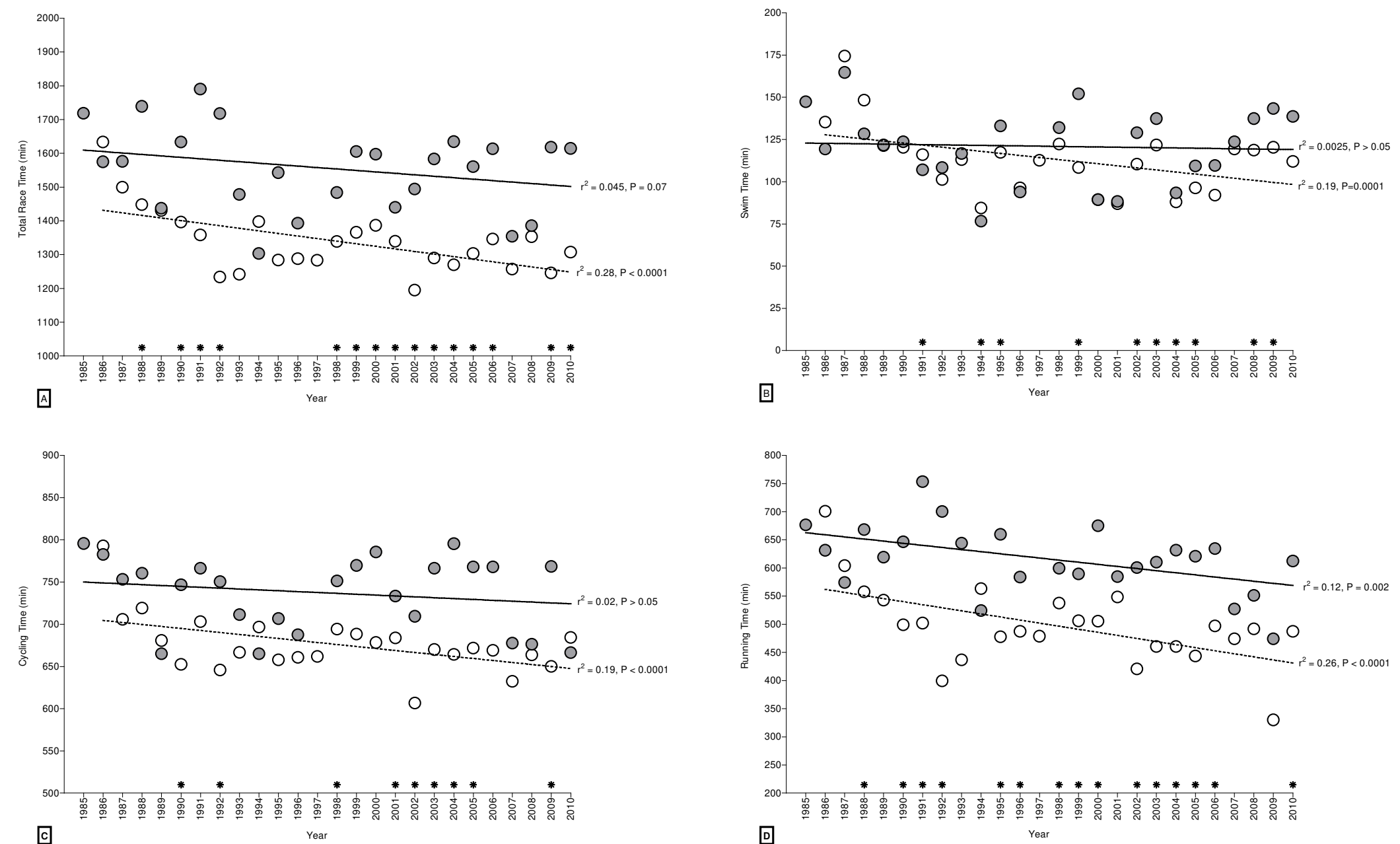

Figure 5 